\section{Prevalência de anemia e deficiência de vitamina A e consumo de ferro e de vitamina A entre crianças usuárias do Sistema Único de Saúde na cidade do Rio de Janeiro, Brasil}

\author{
Prevalence of anemia and vitamin A deficiency \\ and iron and vitamin $A$ intake in children that use \\ the Brazilian Unified National Health System in \\ Rio de Janeiro, Brazil
}

\author{
Prevalencia de anemia, deficiencia de vitamina A, \\ y consumo de hierro y de vitamina $A$ entre niños \\ pacientes del Sistema Único de Salud en la \\ ciudad de Río de Janeiro, Brasil
}

\author{
Inês Rugani Ribeiro de Castro 1 \\ Alessandra da Silva Pereira 1,2 \\ Letícia Barroso Vertulli Carneiro 2 \\ Letícia de Oliveira Cardoso 3 \\ Flávia Fioruci Bezerra 1 \\ Marta Citelli 1 \\ Juliana Martins Oliveira 1 \\ Patricia Afonso Maia 4 \\ Milena Miranda de Moraes 1 \\ Jose Firmino Nogueira Neto 1 \\ Ana Carolina Feldenheimer da Silva 1 \\ Jorginete de Jesus Damião 1
}

doi: 10.1590/0102-311X00252420

\section{Resumo}

Estratégias nacionais para o controle de anemia e deficiência de vitamina A em crianças estão baseadas em estimativas de suas prevalências produzidas em âmbito nacional em 2006 com métodos não validados para este grupo etário e com nível de desagregação para macrorregiões. Com o intuito de subsidiar a gestão local para o (re)direcionamento de medidas de controle desses agravos, o presente trabalho apresenta estimativas de sua prevalência e, também, de marcadores de consumo alimentar de fontes de micronutrientes $e$ do uso de suplementos de vitaminas e minerais em amostra probabilística de crianças de 6 a 59 meses, usuárias da atenção básica de saúde do Município do Rio de Janeiro, Brasil $(n=536)$. Foram coletadas amostras de sangue venoso para análise de hemoglobina, ferritina e retinol sérico e dados sobre o consumo alimentar, o uso de suplementos de vitaminas e minerais e as características sociodemográficas. As prevalências de anemia, anemia ferropriva e deficiência de vitamina A foram de, respectivamente, 13,7\%, 5,5\% e $13 \%$. Quase todas as crianças haviam consumido alimentos ricos em ferro no dia anterior à entrevista, sendo altas as prevalências de consumo de fontes de origem animal. Somente 49,4\% haviam consumido alimentos ricos em vitamina A. As prevalências de uso de algum suplemento, de suplemento com ferro e com vitamina A foram de, respectivamente, 51\%, 14,7\% e 24,4\%. Os resultados apontam a necessidade de redirecionamento das estratégias de prevenção e controle de anemia e deficiência de vitamina A. Estudos futuros são necessários para examinar a evolução desses indicadores, tendo em vista as politicas de austeridade que entraram em vigor nos últimos anos e a crise econômica decorrente da pandemia da COVID-19.

Anemia; Deficiência de Vitamina A; Micronutrientes; Pré-Escolar;

Inquéritos Epidemiológicos
COMUNICAÇÃO BREVE

BRIEF COMMUNICATION 


\section{Introdução}

Mundialmente, a anemia e a deficiência de vitamina A em crianças menores de cinco anos seguem sendo agravos prioritários na agenda da saúde pública. Em 2011, mais de 40\% delas apresentavam anemia 1, e a prevalência de deficiência de vitamina A entre 1995-2005 era superior a 30\% 2.

No Brasil, o Ministério da Saúde desenvolve estratégias para o controle desses agravos 3 com base nos resultados da última Pesquisa Nacional de Demografia e Saúde (PNDS 2006) 4, que apontou prevalências de 20,9\% e 17,4\% para anemia e deficiência de vitamina A, respectivamente, em crianças menores de cinco anos. Na Região Sudeste, as prevalências eram de 22,6\% para anemia e 21,6\% para deficiência de vitamina $\mathrm{A}$, o que, segundo o protocolo do Ministério da Saúde alinhado às diretrizes da Organização Mundial da Saúde (OMS) 5 (prevalência de deficiência de vitamina A superior a 20\%), tornava a região elegível para a ampliação da cobertura do programa de suplementação profilática com megadoses de vitamina A. Entretanto, o desenho amostral da PNDS não permite a desagregação dos resultados em nível municipal ou estadual.

Estudos locais, de diferentes regiões do país, indicam redução de anemia e deficiência de vitamina A em crianças nos últimos 15 anos 6,7,8,9, o que sugere a oportunidade e a necessidade de realização de pesquisas locais em diferentes cenários para se conhecer a situação desses agravos.

O presente estudo foi realizado com o intuito de subsidiar gestores locais na tomada de decisão sobre o (re)direcionamento de medidas para o controle da anemia e da deficiência de vitamina A. São aqui apresentadas as estimativas de prevalência desses agravos e descritos os marcadores de consumo alimentar de fontes de micronutrientes e do uso de suplementos de vitaminas e minerais entre crianças menores de 5 anos, usuárias da atenção básica do Município do Rio de Janeiro, Brasil.

\section{Métodos}

Estudo seccional com amostra probabilística de crianças usuárias do Sistema Único de Saúde (SUS) da cidade do Rio de Janeiro, com idades entre 6 e 59 meses, com seleção em dois estágios (estratificação das unidades básicas de saúde (UBS) por porte e sorteio de crianças destas unidades com base no cadastro de usuários). Foram excluídas as crianças portadoras de doença falciforme ou hepatopatias. Detalhes sobre a amostragem estão disponíveis em Carneiro et al. 10.

Em data pré-agendada, nas UBS sorteadas, pesquisadores treinados coletaram amostra de sangue por punção venosa, mediante jejum superior a quatro horas, e aplicaram aos responsáveis das crianças um questionário com perguntas sobre: caracterização sociodemográfica, acesso a benefício financeiro governamental, segurança alimentar e nutricional em nível domiciliar, uso de suplementos (no momento da pesquisa ou nos quatro meses que a antecederam: uso [sim/não], nome comercial, dose ministrada, periodicidade), aleitamento materno e consumo alimentar (alimentos marcadores de alimentação saudável e não saudável, baseados em listagem do Sistema de Vigilância Alimentar e Nutricional - SISVAN 11, consumidos no dia anterior à pesquisa [sim/não]). Neste estudo, foram consideradas as perguntas referentes a consumo de alimentos fontes de ferro (carnes, ovos, fígado, leguminosas) e de vitamina A (hortaliças e frutas de cor alaranjada, hortaliças folhosas de cor verde escura).

O retinol sérico foi analisado por cromatografia líquida de alta eficiência ${ }^{12}$. A dosagem de hemoglobina foi realizada em aparelho hematológico automatizado (Coulter T890. Beckman Coulter Inc., Estados Unidos). A ferritina foi dosada por turbidimetria/látex. Foram considerados deficiência de vitamina $\mathrm{A}$, anemia e anemia ferropriva valores inferiores aos seguintes pontos de corte, respectivamente: $0,70 \mu \mathrm{mol} / \mathrm{L} 2$ de retinol sérico, $11 \mathrm{~g} / \mathrm{dL}$ de hemoglobina e $12 \mu \mathrm{g} / \mathrm{L}$ de ferritina sérica na presença de anemia 1.

As análises estatísticas foram realizadas no módulo complex samples do programa SPSS versão 19.0 (https://www.ibm.com/), considerando-se o efeito do desenho amostral. Foram calculados os intervalos de 95\% de confiança (IC95\%) para a comparação das frequências das variáveis entre as faixas etárias ( $<24$ e $\geq 24$ meses).

O estudo foi aprovado pelo Comitê de Ética em Pesquisa com Seres Humanos da Secretaria Municipal de Saúde do Rio de Janeiro (SMSRio no 93/13) e financiado pelo Conselho Nacional de 
Desenvolvimento Científico e Tecnológico (CNPq, processos 480804/2013-3 e 420247/2016-5) e pela SMSRio.

\section{Resultados}

As características da amostra estudada $(n=536)$ estão descritas na Tabela 1 . As prevalências de anemia, anemia ferropriva e deficiência de vitamina A foram, respectivamente, 13,7\%, 5,5\% e $13 \%$. As prevalências de anemia e anemia ferropriva foram mais altas entre crianças $<24$ meses quando comparadas às $\geq 24$ meses. Já para a deficiência de vitamina A, não houve diferença estatisticamente significativa entre as faixas etárias (Tabela 2).

Quase todas as crianças haviam consumido alimentos ricos em ferro no dia anterior à entrevista, sendo altas as prevalências de consumo de fontes de origem animal. Por outro lado, somente 49,4\% haviam consumido alimentos ricos em vitamina A (Tabela 3). Os dois grupos analisados apresentaram perfis semelhantes de consumo desses alimentos. As prevalências de uso de algum suplemento e de suplemento com ferro e com vitamina A foram de, respectivamente, 51\%, 14,7\% e 24,4\%, sendo sempre mais altas entre crianças $<24$ meses (Tabela 3 ).

\section{Tabela 1}

Caracterização sociodemográfica da amostra estudada ( $n=536)$. Crianças de 6 a 59 meses de idade usuárias de unidades básicas de saúde. Município do Rio de Janeiro, Brasil, 2014.

\begin{tabular}{|c|c|}
\hline Variáveis & $\%$ \\
\hline \multicolumn{2}{|l|}{ Faixa etária (meses) } \\
\hline$<24$ & 31,8 \\
\hline$\geq 24$ & 68,2 \\
\hline \multicolumn{2}{|l|}{ Sexo } \\
\hline Feminino & 49,8 \\
\hline Masculino & 50,2 \\
\hline \multicolumn{2}{|l|}{ Cor da pele } \\
\hline Branca & 32,3 \\
\hline Parda & 52,0 \\
\hline Preta & 13,3 \\
\hline Amarela/oriental ou Indígena & 2,4 \\
\hline \multicolumn{2}{|l|}{ Nível de insegurança alimentar } \\
\hline Segurança alimentar & 59,7 \\
\hline Insegurança leve & 33,3 \\
\hline Insegurança moderada & 3,9 \\
\hline Insegurança grave & 3,1 \\
\hline \multicolumn{2}{|l|}{ Escolaridade materna } \\
\hline Ensino Fundamental incompleto & 20,4 \\
\hline Ensino Fundamental completo & 34,3 \\
\hline Ensino Médio & 42,0 \\
\hline Ensino Superior & 3,3 \\
\hline \multicolumn{2}{|l|}{ Renda familiar (salários mínimos) } \\
\hline$<0,5$ & 6,0 \\
\hline $0,5-0,9$ & 9,0 \\
\hline $1,0-1,9$ & 46,1 \\
\hline $2,0-2,9$ & 23,2 \\
\hline 3,0 ou mais & 15,7 \\
\hline Recebia benefícios do governo & 37,2 \\
\hline Frequentava creche ou escola & 54,3 \\
\hline
\end{tabular}


Tabela 2

Prevalências de anemia e deficiência de vitamina A segundo faixa etária entre crianças de seis a 59 meses de idade, usuárias de unidades básicas de saúde. Município do Rio de Janeiro Brasil, 2014.

\begin{tabular}{|c|c|c|c|}
\hline \multirow[t]{2}{*}{ Variáveis } & \multicolumn{3}{|c|}{ Prevalência (IC95\%) } \\
\hline & $\begin{array}{c}\text { Total } \\
(n=519) \text { * }\end{array}$ & $\begin{array}{c}<24 \text { meses } \\
(n=188)\end{array}$ & $\begin{array}{c}\geq 24 \text { meses } \\
\quad(n=341)\end{array}$ \\
\hline Anemia & $13,6(10,3-17,9)$ & $28,1(19,9-38,2)$ & $7,0(4,2-11,4)$ \\
\hline Anemia ferropriva & $5,5(3,3-9,0)$ & $12,1(6,5-21,6)$ & $2,5(1,0-6,1)$ \\
\hline Deficiência de vitamina A & $13,0(9,8-17,0)$ & $10,4(6,7-15,9)$ & $14,2(10,0-19,7)$ \\
\hline
\end{tabular}

IC95\%: intervalo de 95\% de confiança.

* Crianças com resultado disponível para as análises bioquímicas.

\section{Tabela 3}

Consumo de alimentos fontes de ferro e de vitamina A e uso de suplementos de micronutrientes entre crianças de seis a 59 meses de idade, usuárias de unidades básicas de saúde. Município do Rio de Janeiro, Brasil, 2014.

\begin{tabular}{|c|c|c|c|}
\hline \multirow[t]{2}{*}{ Indicadores } & \multicolumn{3}{|c|}{ Prevalência (IC95\%) } \\
\hline & $\begin{array}{c}\text { Total } \\
(\mathrm{n}=529)\end{array}$ & $\begin{array}{c}<24 \text { meses } \\
(n=188)\end{array}$ & $\begin{array}{c}\geq 24 \text { meses } \\
(n=341)\end{array}$ \\
\hline \multicolumn{4}{|l|}{ Consumo } \\
\hline Alimentos ricos em ferro & $96,7(94,5-98,1)$ & $95,0(90,9-97,3)$ & $97,5(94,4-98,9)$ \\
\hline Fontes de ferro de origem animal * & $89,5(86,4-92,1)$ & $87,0(81,4-91,1)$ & $90,7(86,6-93,6)$ \\
\hline Fontes de ferro de origem vegetal ** & $86,4(82,6-89,5)$ & $83,0(76,3-88,2)$ & $88,0(83,1-91,6)$ \\
\hline Alimentos ricos em vitamina $\mathrm{A}$ & $49,4(44,2-54,7)$ & $58,8(49,9-67,2)$ & $45,1(38,7-51,7)$ \\
\hline Uso de suplemento de vitaminas e/ou minerais $* \star \star$ & $51,0(45,9-56,0)$ & $72,1(64,2-78,8)$ & $41,5(35,4-47,8)$ \\
\hline Com ferro & $14,7(11,5-18,7)$ & $26,8(19,5-35,5)$ & $9,3(6,3-13,6)$ \\
\hline Com vitamina $\mathrm{A}$ & $24,4(20,4-28,9)$ & $40,0(32,0-48,6)$ & $17,4(13,0-22,8)$ \\
\hline
\end{tabular}

IC95\%: intervalo de 95\% de confiança.

* Carnes, vísceras e ovos;

** Leguminosas e hortaliças verde escuras, como espinafre, taioba, brócolis;

$\star \star \star$ Uso no momento da pesquisa ou nos quatro meses que a antecederam.

\section{Discussão}

As prevalências de anemia e deficiência de vitamina A foram menores do que aquelas estimadas pela PNDS 2006, tanto para o Brasil quanto para a Região Sudeste 4, e corroboram os achados de estudos locais realizados nos últimos 15 anos 6,7,8,9.

Além das diferenças metodológicas entre o nosso estudo e a PNDS 2006, alguns eventos ocorridos entre 2006 e 2014 (ano da coleta de dados) podem explicar, ao menos em parte, esta diferença: o aumento da prática de aleitamento materno exclusivo até os 6 meses de idade e de aleitamento materno entre crianças menores de 24 meses 13, a ampliação da atenção básica à saúde no país e no município estudado 14 e a ampliação da cobertura do Programa Bolsa Família 15. Medidas de fortificação de farinhas não parecem explicar o resultado encontrado 16.

Embora ações de suplementação de ferro possam ter contribuído para essa melhoria, não se pode atribuir a elas o expressivo impacto na realidade estudada, tendo em vista que a cobertura de uso de suplementos com este nutriente era baixa (14,7\%), mesmo entre crianças < 24 meses $(26,8 \%)$, grupo etário mais vulnerável. Por outro lado, embora o programa de suplementação profilática com mega- 
doses de vitamina A não estivesse implantado na rotina das UBS do município estudado, 24,4\% das crianças faziam uso de suplementos que continham este nutriente.

Os indicadores de consumo alimentar sugerem que o consumo de alimentos fonte de ferro pode estar contribuindo para a menor magnitude da anemia. Por outro lado, apontam a importância de se reforçarem ações de Promoção da Alimentação Adequada e Saudável que estimulem o consumo de alimentos ricos em vitamina $\mathrm{A}$.

Este estudo apresenta informações sobre as prevalências de deficiências de micronutrientes baseadas em métodos considerados padrão-ouro para a identificação destes agravos. Além disso, embora não seja representativa de toda a população de crianças moradoras no Rio de Janeiro, a amostra representa as crianças usuárias do SUS neste município, favorecendo a validade externa dos resultados para realidades similares.

A prevalência de deficiência de vitamina A encontrada indica que a implementação da estratégia de suplementação universal de vitamina A não se justificaria no cenário epidemiológico encontrado. Com base nesses achados, essa estratégia não foi implementada.

Os resultados deste estudo indicam a importância de se produzirem evidências atualizadas para a orientação de políticas públicas e de, sempre que possível, produzi-las também em nível local. Estudos futuros são necessários para examinar a evolução dos indicadores aqui descritos, tendo em vista as políticas de austeridade que entraram em vigor nos últimos anos, além da crise econômica decorrente da pandemia da COVID-19.

\section{Colaboradores}

I. R. R. Castro, L. O. Cardoso e J. J. Damião contribuíram com o delineamento do estudo, coordenaram a coleta de dados, supervisionou a análise dos dados e participou da redação do manuscrito. A. S. Pereira, L. B. V. Carneiro, J. M. Oliveira e P. A. Maia contribuíram com o desenho do estudo, participaram da coleta de dados e da construção do banco de dados, conduziram a análise dos dados e redigiu o manuscrito. F. F. Bezerra e M. Citelli contribuíram com o delineamento do estudo e colaboraram nas análises das amostras de sangue e na redação do manuscrito. M. M. Moraes participou da construção do banco de dados, conduziu a análise dos dados e redigiu o manuscrito. J. F. Nogueira Neto contribuiu para o desenho do estudo e coordenou as análises das amostras de sangue. A. C. F. Silva contribuiu com a análise dos dados e participou da redação do manuscrito. Todos os autores leram e aprovaram a versão final para publicação.

\section{Informações adicionais}

ORCID: Inês Rugani Ribeiro de Castro (00000002-7479-4400); Alessandra da Silva Pereira (0000-0002-9382-4724); Letícia Barroso Vertulli Carneiro (0000-0003-0832-2293); Letícia de Oliveira Cardoso (0000-0003-1312-1808); Flávia Fioruci Bezerra (0000-0002-6594-4323); Marta Citelli (0000-0003-1380-3729); Juliana Martins Oliveira (0000-0002-9556-767X); Patricia Afonso Maia (0000-0001-5581-2645); Milena Miranda de Moraes (0000-0002-2652-4051); Jose Firmino Nogueira Neto (0000-0002-5368-5248); Ana Carolina Feldenheimer da Silva (0000-0001-5554-8856); Jorginete de Jesus Damião (0000-0001-6591-3474).

\section{Agradecimentos}

Ao Conselho Nacional de Desenvolvimento Científico e Tecnológico (CNPq 480804/2013-3; 420247/2016-5) e à Secretaria Municipal de Saúde Rio de Janeiro. 


\section{Referências}

1. World Health Organization. The global prevalence of anemia in 2011. Geneva: World Health Organization; 2015.

2. World Health Organization. Global prevalence of vitamin A deficiency in populations at risk 1995-2005. Geneva: World Health Organization; 2009.

3. Secretaria de Atenção à Saúde, Ministério da Saúde. Prevenção e controle de agravos nutricionais. https://aps.saude.gov.br/ape/pcan (acessado em 15/Dez/2020).

4. Ministério da Saúde. Pesquisa Nacional de Demografia e Saúde da Criança e da Mulher PNDS 2006: dimensões do processo reprodutivo e da saúde da criança. Brasília: Ministério da Saúde; 2009. (Série G. Estatística e Informação em Saúde).

5. Departamento de Atenção Básica, Secretaria de Atenção à Saúde, Ministério da Saúde. Manual de condutas gerais do Programa Nacional de Suplementação de Vitamina A. Brasília: Ministério da Saúde; 2013.

6. Ferreira HS, Vieira RCS, Livramento ARS, Dourado BLL, Silva GF, Calheiros MSC. Prevalence of anaemia in Brazilian children in different epidemiological scenarios: an updated meta-analysis. Public Health Nutr 2020; [Online ahead of print].

7. Paula WKAS, Caminha MFC, Figueirôa JN, Batista Filho M. Anemia e deficiência de vitamina A em crianças menores de cinco anos assistidas pela Estratégia Saúde da Família no Estado de Pernambuco, Brasil. Ciênc Saúde Colet 2014; 19:1209-22.

8. Barros ALA, Soares ADN, Pessoa MC, Teixeira RA, Beinner MA. Deficiência de vitamina A em crianças residentes na Região Metropolitana de Belo Horizonte, Minas Gerais. Rev Min Enferm 2010; 14:386-93.

9. Novaes TG, Gomes AT, Silveira KC, Souza CL, Lamounier JA, Netto MP, et al. Prevalência e fatores associados com deficiência de vitamina A em crianças atendidas em creches públicas do Sudoeste da Bahia. Rev Bras Saúde Matern Infant 2016; 16:337-44.
10. Carneiro LBV, Castro IRR, Juvanhol LL, Gomes FS, Cardoso LO. Associação entre insegurança alimentar e níveis de hemoglobina e retinol em crianças assistidas pelo Sistema Único de Saúde no Município do Rio de Janeiro, Brasil. Cad Saúde Pública 2019; 36:e00243418.

11. Ministério da Saúde. Orientações para avaliação de marcadores de consumo alimentar na atenção básica. 2a Ed. Brasília: Ministério da Saúde; 2016.

12. Hess D, Keller HE, Oberlin B, Bonfanti R, Schüep W. Simultaneous determination of retinol, tocopherols, carotenes and lycopene in plasma by means of high-performance liquid chromatography on reversed phase. Int $\mathrm{J} \mathrm{Vi-}$ tam Nutr Res 1991; 61:232-8.

13. Universidade Federal do Rio de Janeiro. Estudo Nacional de Alimentação e Nutrição Infantil - ENANI-2019: resultados preliminares. Indicadores de aleitamento materno no Brasil. Rio de Janeiro: Universidade Federal do Rio de Janeiro; 2020.

14. Ferreira de Souza PH, Osorio RG, Paiva LH, Soares S. Os efeitos do Programa Bolsa Família sobre a pobreza e desigualdade: um balanço dos primeiros quinze anos. Rio Janeiro: Instituto de Pesquisa Econômica Aplicada; 2019.

15. Ramos ALP, De Seta MH. Atenção primária à saúde e Organizações Sociais nas capitais da Região Sudeste do Brasil: 2009 e 2014. Cad Saúde Pública 2019; 35:e00089118.

16. Formoso AMC, Santos IS, Barros AJD, Gigante DP, Victora CG. Efeito da fortificação de farinhas com ferro sobre anemia em pré-escolares, Pelotas - RS. Rev Saúde Pública 2007; 41:53948. 


\begin{abstract}
Brazilian national strategies for the control of anemia and vitamin A deficiency in children are based on estimates of their nationwide prevalence rates in 2006 with methods not validated for this age group and with disaggregation at the level of major geographic regions. To back local administrations in (re)directing control measures for these two disorders, the current study presents estimates of their prevalence and markers of dietary intake of sources of micronutrients and use of vitamin and mineral supplements in a probabilistic sample of children 6 to 59 months of age, users of primary healthcare in the city of Rio de Janeiro, Brazil $(n=536)$. Venous blood samples were drawn for analysis of hemoglobin, ferritin, and serum retinol, besides collection of data on food consumption, use of vitamin and mineral supplements, and sociodemographic characteristics. Prevalence rates for anemia, iron deficiency anemia, and vitamin A deficiency were $13.7 \%, 5.5 \%$, and $13 \%$, respectively. Nearly all the children had consumed ironrich food the day before the interview, with high prevalence of animal sources. Only $49.4 \%$ had consumed foods high in vitamin $A$. The prevalence rates for use of any supplement, iron supplements, and vitamin A supplements were $51 \%, 14.7 \%$, and $24.4 \%$, respectively. The findings point to the need to redirect the strategies for prevention and control of anemia and vitamin A deficiency. Future studies are necessary to examine trends in these indicators, focusing on austerity policies implemented in recent years and the economic crisis resulting from the COVID-19 pandemic.
\end{abstract}

Anemia; Vitamina A Deficiency; Micronutrients; Preschool Child; Health Surveys

\section{Resumen}

Las estrategias brasileñas para el control de anemia y deficiencia de vitamina $A$ en niños están basadas en estimaciones de sus prevalencias, producidas en el ámbito nacional en 2006 con métodos no validados para este grupo etario, $y$ con un nivel de desagregación en las macrorregiones. Con el fin de apoyar la gestión local para la (re)orientación de medidas de control de esos problemas de salud, este trabajo presenta estimaciones de su prevalencia y, también, de los marcadores de consumo alimentario de fuentes de micronutrientes $y$ del uso de suplementos de vitaminas y minerales, en una muestra probabilística de niños de 6 a 59 meses, pacientes de atención básica de salud del Municipio de Río de Janeiro, Brasil $(n=536)$. Se recogieron muestras de sangre venosa para el análisis de hemoglobina, ferritina y retinol sérico, así como datos sobre el consumo alimentario, de suplementos de vitaminas y minerales, así como de características sociodemográficas. Las prevalencias de anemia, anemia ferropénica y deficiencia de vitamina A fueron de, respectivamente, 13,7\%, 5,5\% y 13\%. Casi todos los niños habian consumido alimentos ricos en hierro el día anterior a la entrevista, siendo altas las prevalencias de consumo de fuentes de origen animal. Solamente un 49,4\% habian consumido alimentos ricos en vitamina $A$. Las prevalencias de consumo de algún suplemento, de suplemento con hierro y de suplemento con vitamina A fueron de, respectivamente, 51\%, 14,7\% y 24,4\%. Los resultados apuntan la necesidad de reorientar las estrategias de prevención y control de la anemia y deficiencia de vitamina A. Se necesitan estudios futuros para examinar la evolución de esos indicadores, teniendo en vista las políticas de austeridad que entraron en vigor en los últimos años y la crisis económica a consecuencia de la pandemia de COVID-19.

Anemia; Deficiencia de Vitamina A; Micronutrientes; Preescolar; Encuestas Epidemiológicas
Recebido em 25/Ago/2020

Versão final reapresentada em 16/Dez/2020

Aprovado em 04/Mar/2021 